\title{
Umsetzung der aktuellen S3-Leitlinie zur Prävention, Diagnostik, Therapie und Nachsorge des nicht-kleinzelligen Lungenkarzinoms am Diakoniekrankenhaus Halle/Saale
}

\author{
Implementation of the German Guideline for the Prevention, Diagnosis, Treatment and Follow-up of \\ Non-small Cell Lung Cancer at the Diakoniekrankenhaus Halle/Saale
}

Autoren

Institute
M. Witzmann' ${ }^{1}$ K.-P. Litwinenko' ${ }^{1}$, O. Fischbeck ${ }^{1}$, W. Schütte ${ }^{2}$

Klinik für Pneumologie, Thoraxchirurgie und Palliativmedizin, Diakoniekrankenhaus, Halle/Saale

Klinik für Innere Medizin II, Krankenhaus Martha-Maria Halle-Dölau, Halle/Saale eingereicht 19.8.2013 akzeptiert nach Revision 17.9.2013

\section{Bibliografie}

Dol http://dx.doi.org/ $10.1055 / \mathrm{s}-0033-1344885$ Online-Publikation: 12.11 .2013 Pneumologie 2013; 67: 683-687 (c) Georg Thieme Verlag KG Stuttgart $\cdot$ New York ISSN 0934-8387

Korrespondenzadresse Mathias Witzmann Weidenplan 19 06108 Halle/Saale m.witzmann@gmx.net

\section{Zusammenfassung \\ $\nabla$}

Hintergrund: Mehr als 3 Jahre nach Veröffentlichung der S3-Leitlinie zur Diagnostik und Therapie des Lungenkarzinoms ist die Datenlage zum Umsetzungsgrad von Leitlinienempfehlungen unzureichend. Ziel dieser Arbeit ist die Analyse der Umsetzung der empfohlenen Algorithmen zur First-Line-Therapie.

Patienten und Methoden: Es wurden retrospektiv sämtliche Patienten ermittelt, die im Zeitraum Januar 2010 bis Dezember 2011 mit der Erstdiagnose eines nicht-kleinzelligen Lungenkarzinoms am Diakoniekrankenhaus Halle/Saale eine FirstLine-Therapie erhielten. Anhand der Krankenakten wurden demographische Daten, Tumorstadium, Zeitpunkt der Erstdiagnose sowie Daten zur Erstlinientherapie ermittelt. Die Einordnung der Patienten in die Gruppen „leitliniengerechte Behandlung“ und „von der Leitlinie abweichende Behandlung“ erfolgte stadienabhängig anhand der jeweiligen Algorithmen aus dem Leitlinienkapitel „Therapie des nicht-kleinzelligen Lungenkarzinoms“.

Ergebnisse: 126 von 148 untersuchten Fällen (85\%) wurden den Leitlinienempfehlungen entsprechend therapiert. 22 Fälle (15\%) erhielten eine von den Empfehlungen abweichende Erstlinientherapie. Als Begründungen für abweichende Therapieschemata konnten die Untergruppen „Reduzierter Allgemeinzustand“, „Technische Faktoren“, „Patientenentscheidung“ sowie „Sonstige" ermittelt werden.

Schlussfolgerungen: Bezogen auf das analysierte Patientenkollektiv besteht mit $85 \%$ ein starker Durchsetzungsgrad der Leitlinienempfehlungen im Beobachtungszeitraum. Andererseits zeigt die Studie, dass nicht jeder individuelle Fall der komplexen Lungenkrebserkrankung durch die Leitlinie abgebildet werden kann. Diese Tatsache bedingt ein ausgeprägt heterogenes Begründungsspektrum für das Abweichen von Therapieemp-

\section{Abstract \\ $\nabla$}

Background: More than three years since the release of the german guideline for the prevention, diagnosis, treatment and follow-up of lung cancer the database in terms of the implementation of guideline recommendations is deficient. The aim of this article is to analyze the implementation of the recommended algorithms for first-line therapy of non-small cell lung cancer.

Patients and Methods: On the basis of the patients records we determined all cases of newly diagnosed non-small cell lung cancer which received a first-line therapy at the Diakoniekrankenhaus Halle/Saale between January 2010 and December 2011. The demographic data, tumor stage, time of diagnosis and performed first-line therapy were documented. Each case was assigned to the groups "guideline-adherent treatment" or "deviation from guideline recommendation" in dependency of its tumor stage. For this assignment the corresponding algorithms from guideline chapter "Therapy of non-small lung cancer" were used. Results: A total of 126 from 148 cases (85\%) received guideline-adherent treatment. Deviation from guideline recommendation was found in 22 cases (15\%). The categories "poor performance status", "technical factors", "patient decision" and "others" were determined as the main reasons for non guideline-adherent treatment.

Conclusions: Based on the analyzed population this study determined a high grade of guideline adherence at the period of investigation. Otherwise it shows that guideline recommendations cannot include each individual factor of the complex lung cancer disease. It could be found a wide range of reasons for deviation from the guideline recommendations. 
fehlungen. Zukünftig sind weiterführende Studien und Analysen vor allem zur Entwicklung und Optimierung hausinterner Standards zur Behandlungsqualität bezüglich der geltenden Leitlinien notwendig.

\section{Einleitung}

Mit der im Februar 2010 publizierten S3-Leitlinie zur Prävention, Diagnostik, Therapie und Nachsorge des Lungenkarzinoms ist durch das Mitwirken zahlreicher Fachgesellschaften eine auf hohem Evidenzlevel basierende Entscheidungshilfe vor allem für das interdisziplinäre Management der komplexen Erkrankung Lungenkrebs entstanden [1]. Jedoch ist die Datenlage zur Versorgung von Lungenkrebspatienten in Deutschland insgesamt unzureichend [2]. International existieren einige Untersuchungen zur Umsetzung von Leitlinien, in denen einzelne Therapiemodalitäten [3-5] bzw. isolierte Tumorstadien betrachtet werden [6,7]. Die Analyse der Umsetzung von Leitlinienempfehlungen in Deutschland erfolgte erstmalig in Form einer Querschnittserhebung für das Bundesland Berlin. Diese Arbeit postuliert eine nahezu vollständige konzeptionelle Leitlinientreue in stationären pneumologischen bzw. pneumologisch-thoraxchirurgischen Einrichtungen [8]. Zum weiteren Erkenntnisgewinn bezüglich dieser Problematik soll unsere Studie durch die Analyse der Leitlinientreue bei der First-Line-Therapie von Patienten mit nicht-kleinzelligem Bronchialkarzinom beitragen. Die Arbeit fokussiert explizit nur die angewandten First-Line-Therapieregime. Auf die Auswertung von Diagnostik, Second-Line- sowie weiteren Folgetherapien wurde gezielt verzichtet. Die Datenerfassung erfolgte retrospektiv über einen 2-Jahres-Zeitraum an einem spezialisierten Zentrum in Halle/Saale.

\section{Patienten und Methoden}

Die Datenanalyse erfolgte retrospektiv anhand von Krankenakten sowie Protokollen des hausinternen Tumorboards. In die Untersuchung eingeschlossen wurden konsekutiv alle Patienten mit der Erstdiagnose eines nicht-kleinzelligen Lungenkarzinoms, die im Zeitraum Januar 2010 bis Dezember 2011 am Diakoniekrankenhaus Halle/Saale, einer Klinik der Basisversorgung mit TÜVzertifiziertem Lungenkrebszentrum (ISO 9001:2008), eine FirstLine-Therapie erhielten. Sämtliche eingeschlossenen Fälle wurden initial im interdisziplinären Tumorboard der Klinik (bestehend aus den Fachdisziplinen Radiologie, Strahlentherapie, Nuklearmedizin, Thoraxchirurgie sowie Innere Medizin) besprochen. Die getroffenen Entscheidungen waren entsprechend schriftlich protokolliert und elektronisch erfasst.

Insgesamt befanden sich im Rekrutierungszeitraum 190 Patienten mit einem Lungenkarzinom jeglicher Histologie in Behandlung. Hiervon fanden 38 Patienten keine Berücksichtigung, bei denen eine abweichende histologische bzw. zytologische Diagnose festgestellt wurde, darunter 31 kleinzellige, 4 mischzellige Tumore sowie 3 Karzinoidtumore. Ein unvollständiger Datenbestand im Sinne der Einschlusskriterien (Sicherung der Erstdiagnose im Zeitraum Januar 2010 bis Dezember 2011 sowie histologische/zytologische Sicherung eines nicht-kleinzelligen Karzinoms) war zu eruieren in insgesamt 4 Fällen. In Zusammenschau dieser nicht berücksichtigten Fälle gingen somit die Daten von 148 Patienten in die Untersuchung ein.
Als patientenbezogene Daten wurden die Variablen Alter, Geschlecht, Datum der Erstdiagnose, Tumorhistologie (TNM 7.Aufl.), Allgemeinzustand (ECOG-Performance-Status) und Komorbidität erfasst. Bezüglich der Erstlinientherapie wurden die Modalitäten Operation, Radiatio, Chemotherapie und best supportive care erfasst. Das mittlere Alter des Gesamtkollektivs betrug 67 Jahre. In der Geschlechtsverteilung überwog das männliche Geschlecht deutlich (72\% Männer; 28\% Frauen). Der größte Anteil der Patienten (68\%) wies einen initialen ECOG Performance Status von 1 auf. Die diagnostizierten Tumorstadien umfassten die Stadien I - IV, wobei mit 37\% der größte Anteil der Fälle bereits ein metastasiertes Tumorstadium aufwies. Mehr als die Hälfte der Patienten (58\%) wurde initial mit einer Monotherapie (alleinige Operation bzw. Radio- oder Chemotherapie) behandelt ( Tab. 1).

Zur weitergehenden Analyse der Leitlinientreue wurden die rekrutierten Fälle den Gruppen „leitliniengerechte Behandlung“ oder „von der Leitlinie abweichende Behandlung“ zugeordnet. Die Zuordnung erfolgte stadienabhängig anhand der jeweiligen Algorithmen aus dem Leitlinienkapitel „Therapie des nicht-kleinzelligen Lungenkarzinoms“. Um eine zweifelsfreie und nachvollziehbare Einordnung zu gewährleisten, analysierten wir für jeden einzelnen Fall getrennt, ob der jeweilige (für das initiale Tumorstadium empfohlene) Algorithmus für die First-Line-Therapie bis zum Ende verfolgt wurde. War dies nicht der Fall, so wurde der betreffende Fall als „von der Leitlinie abweichend behandelt" gewertet und die entsprechende Begründung für ein abweichendes Therapieregime dokumentiert. Zur Systematisierung und besseren Auswertbarkeit wurden bezüglich des Begründungsspektrums für abweichende Therapieregime 4 Subgruppen gebildet $($ Tab.2).

Tab. 1 Charakteristika des Gesamtkollektivs

\begin{tabular}{|lll|}
\hline Anzahl gültige Fälle & 148 & \\
\hline Mittleres Alter bei ED [Jahre ] & $67(34-88)$ & \\
\hline $\begin{array}{l}\text { Geschlecht } \\
\mathrm{n}(\%)\end{array}$ & männlich & weiblich \\
\hline & $107(72)$ & $41(28)$ \\
\hline & & $n(\%)$ \\
\hline ECOG & 0 & $9(6)$ \\
& 1 & $101(68)$ \\
& 2 & $27(18)$ \\
& 3 & $8(6)$ \\
& 4 & $3(2)$ \\
\hline Tumorstadium & & $n(\%)$ \\
& I & $39(26)$ \\
& II & $20(14)$ \\
\hline & III & $34(23)$ \\
\hline Monotherapie & IV & $55(37)$ \\
Multimodale Therapie & & $8(\%)$ \\
\hline Best supportive care & & $53(58)$ \\
\hline
\end{tabular}

ED: Erstdiagnose.

Tab. 2 Begründungskategorien für abweichende Therapieregime.

\section{Bezeichnung der Kategorie}

Reduzierter Allgemeinzustand

Technische Faktoren

Patientenentscheidung

Sonstige 


\section{Ergebnisse}

In 126 von 148 untersuchten Fällen (85\%) konnte eine leitliniengerecht durchgeführte First-Line-Therapie festgestellt werden. In 22 Fällen (15\%) wurden Abweichungen von den Therapieempfehlungen gefunden ( $\bullet$ Abb. 1). Zur Methodik sei an dieser Stelle erwähnt, dass als „von der Leitlinie abweichend behandelt“ auch jene Fälle definiert waren, die aus eigenem Willen Therapiemaßnahmen ablehnten (Kategorie „Patientenentscheidung“). Der Einschluss dieser Patienten erfolgte aufgrund der Tatsache, dass in den 10 benannten Fällen Maßnahmen abgelehnt wurden, die zuvor im Tumorboard entsprechend der Leitlinienvorgaben beschlossen wurden. Somit konnte auch in diesen Fällen keine empfohlene First-Line-Therapie durchgeführt werden. Tab.3 zeigt eine zusätzliche Übersicht über die Charakteristika dieser Subgruppe.

- Tab. 4 stellt systematisch die Verteilung der festgestellten Abweichungen (stadienabhängig) auf die verschiedenen Begründungskategorien dar.

Innerhalb der Kategorien „Reduzierter Allgemeinzustand“ und „Technische Faktoren“ resultierten alle festgestellten Abweichungen von den Therapieempfehlungen aus Einzelfallentscheidungen: Drei Fälle im Stadium IA wurden initial als operabel eingestuft, intraoperativ jedoch wurde letztlich aufgrund einer ausgeprägten pulmonalen Funktionseinschränkung und des resultierenden schlechten Leistungsstatus auf die für alle operierten Patienten empfohlene mediastinale Lymphknotendissektion (und somit auf einen onkologisch radikalen Eingriff) verzichtet. In drei Fällen (Stadium IIB) erfolgte initial eine in diesem Stadium nicht empfohlene neoadjuvante Radiochemotherapie. Hierbei handelte es sich um technisch schwierig zu resezierende Tumo-

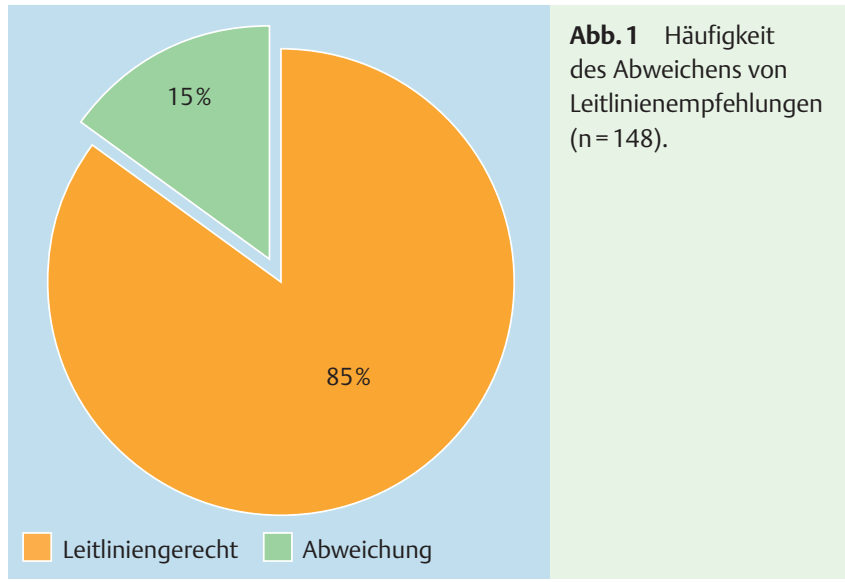

ren, sodass sich interdisziplinär für dieses individuelle Vorgehen entschieden wurde, um zunächst eine Tumorverkleinerung zu erreichen. In drei weiteren Fällen (Stadium IIIB) wurde trotz eines initialen ECOG von 1 auf die für dieses Stadium empfohlene kombinierte Radiochemotherapie verzichtet und lediglich eine Chemotherapie durchgeführt. Auch hier erfolgte eine individuelle Entscheidung für diese Vorgehensweise aufgrund des klinischen Gesamtbildes der Patienten, welches trotz definitionsgemäß gutem Allgemeinzustand eine großräumige Radiotherapie als nicht tolerabel erscheinen ließ. Die Kategorie „Sonstige“ umfasst jene Fälle $(n=3)$, bei denen keine definitive Begründung für eine abweichende First-Line-Therapie nachvollzogen werden konnte. Abb. 2 gibt einen Überblick über die prozentuale Verteilung der verschiedenen Begründungskategorien.

\begin{tabular}{|c|c|c|c|}
\hline$n=10$ & & & \\
\hline Mittleres Alter bei ED [Jahre] & $73,5(51-88)$ & & \\
\hline Geschlecht & männlich & weiblich & \\
\hline$n(\%)$ & $6(60)$ & $4(40)$ & \\
\hline & & $n(\%)$ & \\
\hline & 0 & - & \\
\hline & 1 & $5(50)$ & \\
\hline ECOG & 2 & $5(50)$ & \\
\hline & 3 & - & \\
\hline & 4 & - & \\
\hline & & $n(\%)$ & Definition der Abweichung \\
\hline & I & $3(30)$ & keine systematische MLKD \\
\hline & II & $2(20)$ & keine adjuvante $\mathrm{CT}$ bei $\mathrm{pN} 1$ postoperativ \\
\hline Tumorstadium & IIIA & $1(10)$ & keine kombinierte RCT bei IIIA4 \\
\hline & IIIB & - & - \\
\hline & $\mathrm{IV}^{1}$ & $4(50)$ & keine Chemotherapie \\
\hline
\end{tabular}

Tab. 3 Charakteristika der Subgruppe „Patientenentscheidung“.

MLKD: Mediastinale Lymphknotendissektion; CT: Chemotherapie; RCT: Radiochemotherapie.

${ }^{1}$ Aufgrund Patientenentscheidung keine EGFR-Mutationsanalyse durchgeführt.

Tab. 4 Stadienabhängige Abweichungen.

\begin{tabular}{|c|c|c|c|c|c|}
\hline Stadium & $\mathbf{I}$ & II & IIIA & IIIB & IV \\
\hline n (gesamt) & 39 & 20 & 16 & 18 & 55 \\
\hline n (Abweichung) & 3 & 4 & - & 3 & 2 \\
\hline Kategorie & $\begin{array}{l}\text { reduzierter } \\
\text { Allgemeinzustand }\end{array}$ & $\begin{array}{l}\text { technische Faktoren/ } \\
\text { sonstige }\end{array}$ & - & technische Faktoren & sonstige \\
\hline & keine systematische & neoadjuvante & & keine kombinierte & \\
\hline Definition der Abweichung & MLKD & Radiochemotherapie & - & RCT & - \\
\hline Patientenentscheidung (n) & 3 & 2 & 1 & - & 4 \\
\hline
\end{tabular}

MLKD: Mediastinale Lymphknotendissektion; RCT: Radiochemotherapie. 


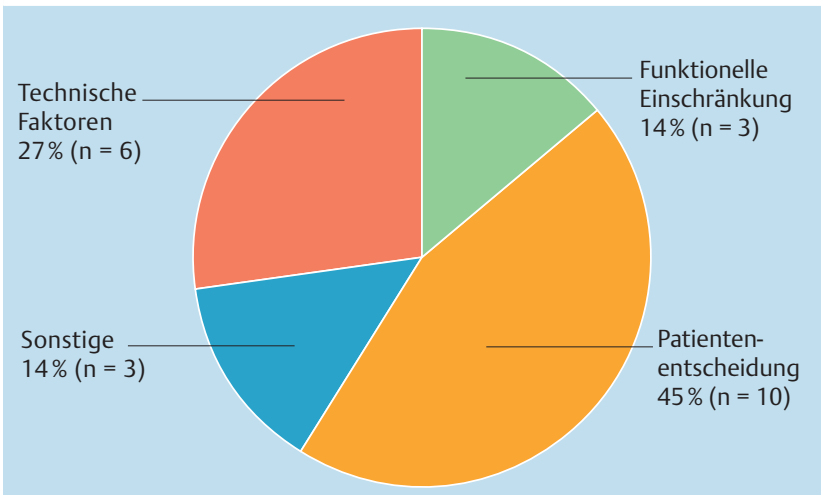

Abb.2 Prozentuale Verteilung der Begründungskategorien (Von der Leitlinie abweichend behandelte Fälle; $n=22$ ).

\section{Diskussion}

$\nabla$

Bezüglich der Umsetzung internationaler Leitlinien zum NSCLC liegen Analysen aus Datenbanken mit großen Patientenzahlen vor. So fanden Wang et al. in ihrer retrospektiven Analyse Umsetzungsgrade zwischen 61,3\% bzw. 75\% [5]. Diese Studie betrachtete die Umsetzung der Empfehlungen zur Chemotherapie in frühen sowie metastasierten Tumorstadien. Zornosa et al. analysierten mit $76 \%$ eine ähnliche Umsetzungsrate, wobei hier lediglich metastasierte Tumorstadien betrachtet wurden [6]. Ein umfassenderes Spektrum betrachteten Vinod et al. in einer prospektiven Studie, in der alle Tumorstadien vertreten waren. Hier wurde eine leitliniengerechte Therapie für $71 \%$ der Fälle beschrieben [9].

Für Leitlinien aus dem deutschsprachigen Raum existieren zum Durchsetzungsgrad wenige valide Daten aus systematischen Analysen. Daher beabsichtigen wir mit unserer Studie zur aktuellen S3-Leitlinie zur Verbesserung der bestehenden Datenlage beizutragen. Anhand der in unserer Analyse herausgefilterten Übereinstimmung bezüglich der First-Line-Therapie von 85\% können wir die von Blum et al. [8] festgestellte hochgradige Leitlinientreue für stationäre Einrichtungen bestätigen. Als methodischer Unterschied muss hier das monozentrische Studiendesign unserer Arbeit gesehen werden, welches die schrittweise Analyse der einzelnen Algorithmen vorsieht, ohne wie Blum et al. anhand von Fragebögen explizit auf die in der Leitlinie publizierten Qualitätsindikatoren einzugehen. Außerdem beschränkt sich die Untersuchung nicht wie einige der vorgenannten Studien lediglich auf einzelne Therapiemodalitäten oder Tumorstadien, sondern schließt sämtliche im Untersuchungszeitraum vorhandenen Therapieregime und Tumorentitäten ein. Dies stellt einen möglichen Einflussfaktor auf die Übereinstimmungsrate dar.

Weiterhin stellen sich folgende Limitationen dar: In unserer Arbeit wird die Umsetzung der Leitlinie durch das "hauseigene“ Team thematisiert, worauf an dieser Stelle als mögliche methodische Einschränkung hingewiesen werden soll. Aufgrund des retrospektiven Charakters der Analyse und der damit verbundenen Auswertung von Therapieentscheidungen aus der Vergangenheit sind Informationsverluste und somit eine Verzerrung der Einordnung der Entscheidungen möglich. Ein wichtiger möglicher Störfaktor ist das Auftreten von in der Leitlinie nicht vollständig abgebildeten Konstellationen, vor allem für Patienten in den Stadien IIIA/IIIB (mögliche multimodale Therapiekonzepte, z.B. Radio-/Chemotherapie simultan oder sequenziell). In diesen Fällen sind Verschiebungen in der Interpretation der Adhärenz zu den Empfehlungen möglich. Des Weiteren ist der relativ hohe Anteil der Patienten in Stadium I/II und das damit verbundene erniedrigte Durchschnittsalter als möglicher Einflussfaktor zu nennen; zudem befinden sich in der analysierten Population mehr Männer als im bundesdeutschen Durchschnitt.

Bezüglich der Begründungen für abweichende Therapieentscheidungen zeigt die Studie ein ausgeprägt heterogenes Spektrum, welches vorrangig von individuellen bzw. technischen Faktoren abhängt. Diese Ergebnisse lassen die Schlussfolgerung zu, dass, wie bereits angedeutet, durch die Leitlinie nicht jeder individuelle Erkrankungsfall abgebildet wird, was den Stellenwert der interdisziplinären Therapieentscheidung im Sinne der Qualitätssicherung der Behandlung umso mehr hervorhebt. Bezüglich dieser Feststellung konnten in einer Untersuchung von Osarogiagbon et al. für Patienten, die in interdisziplinären Tumorboards besprochen und entsprechend der Empfehlungen therapiert wurden, bereits Vorteile hinsichtlich des Gesamt- und Progressionsfreien Überlebens nachgewiesen werden [10].

Aus den erhobenen Daten der von uns untersuchten Population lassen sich einige Ansätze ableiten, die zu einer Optimierung der Adhärenz zu den Leitlinienempfehlungen führen können. So ist, auch in kleineren regionalen Einrichtungen mit onkologischpneumologischem Schwerpunkt, die Besprechung der Fälle durch ein interdisziplinäres Tumorboard unerlässlich, um eine adäquate und umfassende Analyse jedes einzelnen Erkrankungsfalles zu gewährleisten. Die entsprechende korrekte Einordnung der Patienten in den richtigen therapeutischen Algorithmus anhand von Staging und Allgemeinzustand sowie weiterer (individueller) Erkrankungsfaktoren kann nur durch eine solche fachübergreifende Zusammenarbeit erreicht werden. Ferner muss vor allem bei Konstellationen, die multimodale Therapiekonzepte erlauben (z. B. Patienten in Stadium IIIA/IIIB) oder in Fällen, bei denen die „Therapiefähigkeit“ des Patienten in Frage steht, eine sorgfältige Evaluierung der therapeutischen Möglichkeiten anhand der vorgegebenen Therapiepfade erfolgen.

Ein Ansatz zur qualitativen Verbesserung von Entscheidungsprozessen wäre eine regelmäßige (retrospektive) hausinterne Evaluierung der behandelten Fälle, um z.B. Erkrankungskonstellationen zu identifizieren, die häufig zu nicht-leitliniengerechten Therapieentscheidungen führen, und somit entsprechende Lösungsansätze zu entwickeln.

In Zusammenschau der Ergebnisse besteht also weiterhin, vor allem hinsichtlich der Analyse der Umsetzungsraten von Leitlinien sowie der Gründe für mangelnde Leitlinientreue, weiterer Bedarf besonders an prospektiven Studien mit ausreichenden Patientenzahlen, um ein differenzierteres Bild zu diesen Fragestellungen zu erhalten und somit eine weitere Verbesserung der Übereinstimmungsraten zu erzielen.

\section{Interessenkonflikt}

Die Autoren geben an, dass kein Interessenkonflikt besteht. 


\section{Literatur}

1 Goeckenjan G, Sitter H, Thomas $M$ et al. Prävention, Diagnostik, Therapie und Nachsorge des Lungenkarzinoms. Pneumologie 2010; 64: e1 164

2 Blum T, Schönfeld N, Kollmeier J et al. Lungenkrebs in Deutschland - zur Versorgungslage der Nation. Pneumologie 2011; 65: 7-18

3 Heijmen L, van Laarhoven HWM, Punt CJA et al. Encouraging results in older patients receiving chemotherapy: a retrospective analysis of treatment guideline adherence in daily practice. Geriatr Gerontol Int 2012; $12: 80-85$

4 Salloum RG, Smith TJ, Jensen GA et al. Factors associated with adherence to chemotherapy guidelines in patients with non-small cell lung cancer. Lung Cancer 2012; 75: 255-260

5 Wang Z, Askamit I, Tuscher $L$ et al. Rates of guideline adherence among US community oncologists treating NSCLC. Am J Manag Care 2013; 19: $185-192$
6 Zornosa C, Vandergrift JL, Kalemkerian GP et al. First-line systemic therapy practice patterns and concordance with $\mathrm{NCCN}$ guidelines for patients diagnosed with metastatic NSCLC treated at NCCN institutions. J Natl Compr Canc Netw 2012; 10: 847-856

7 Gridelli C, Ardizzoni A, Barni S et al. Medical treatment choices for patients affected by advanced NSCLC in routine clinical practice. Results from the Italian observational "SUN" (Survey on the lUng cancer maNagement) study. Lung cancer 2011; 74: 462-468

8 Blum T, Schönfeld N, Goeckenjan G et al. Umsetzung der S3-Leitlinie zur Prävention, Diagnostik, Therapie und Nachsorge des Lungenkarzinoms im Land Berlin. Pneumologie 2013; 67: 118-122

9 Vinod SK, Sidhom MA, Delaney GP. Do multidisciplinary meetings follow guideline-based care? J Oncol Pract 2010; 6: 276-281

10 Osarogiagbon RU, Phelps G, McFarlane J et al. Causes and consequences of deviation from multidisciplinary care in thoracic oncology. J Thorac Oncol 2011; 6: 510-516 